\title{
Genome Integrity and Organization in the Context of Radiobiology
}

\author{
Vladan Ondřej \\ Laboratory of Genome Integrity, Faculty of Science, \\ Palacký University in Olomouc, \\ Czech Republic
}

\section{Introduction}

The genomes of all organisms on Earth are exposed to various stimuli that induce DNA damage in the form of single- or double-stranded DNA breaks. One of the stimuli that impair genome stability is the irradiation of cells by ionizing radiation. Organisms have mechanisms to prevent the disruption of genome integrity by DNA breaks. The repair of double-stranded DNA breaks (DSBs) is mediated by two major pathways: nonhomologous recombination (NHEJ), and homologous recombination repair (HRR). However, a lot of the studies are focused on the repair pathways, the DNA repair must also be studied in the context of genome organization. Genomes are organized into complex higher-order structures by folding of the DNA into chromatin fibres, loops, domains, and chromosome territories. This functional organization is important, not only for gene regulation and control of gene expression patterns; but further, they also provide an environment for DNA repair processes. Defects in higher-order chromatin structures affects DNA repair, making cells more sensitive to ionizing radiation, and they are relevant in many physiological and pathological processes. An understanding of the global higher-order chromatin structures also shines a light into those processes causing chromosomal abnormalities, such as translocations, which are a common hallmark of cancer cells.

\section{Higher-order chromatin structure and DSB repair}

Most of the genome of Eukaryotes is deposed within the cell nucleus, with the remainder in the mitochondria (in the case of plants, in the chloroplasts). The cell nucleus provides not only the space and environment for the genome, but also the organization and machinery required for genomic function and maintenance. Nuclear architecture is not a rigid structure just for DNA deposition, as was thought for many decades prior. Rather, it is a dynamic and highly organized organelle, reflecting all of the requirements for managing the genetic data such as replication and transcription; additionally, this includes DNA repair.

\subsection{Chromatin structure}

In their native state, genomes are folded into complex higher-order structures that interact with nuclear proteins, as well as protein complexes at the nuclear periphery, and they 
communicate with the cytoplasm through the nuclear pores. At the lowest level, the DNA is wrapped around an octamer of core histone proteins (which are the primary structural elements of the chromatin fibre) in order to form a nucleosome. Multiple nucleosomes are linked by stretches of DNA, often occupied by a linker histone, into a 'beads-on-a string' fibre of $10 \mathrm{~nm}$ in diameter. These strings of linked nucleosomes are helically twisted into a $10 \mathrm{~nm}$ fibre, which in turn is folded into a $30 \mathrm{~nm}$ fibre. There are indications that this fibre is folded into yet higher order chromatin loops, although the topological organization of these higher order structures in vivo is unclear (reviewed in Misteli, 2010). In some models, the compaction of chromatin is linked to the size of the loops, and distinguishes the chromatin from the compact heterochromatin and decondensed euchromatin (Goetze et al., 2007).

\subsection{Epigenetic modifications of the chromatin}

Chromatin, the physiological template of all eukaryotic genetic information, is subject to diverse epigenetic modifications of the DNA and histones. Large arrays of posttranslational modifications of the histones, which largely impinge on the histone amino termini, regulate access to the underlying DNA. Distinct histone amino-terminal modifications can generate synergistic or antagonistic interaction affinities for chromatin-associated proteins; in turn, these dictate the dynamic transitions between transcriptionally active or transcriptionally silent chromatin states. Thus, the combinatorial nature of histone amino-terminal modifications reveals a "histone code" that considerably extends the information potential of the genetic code (Jenuwain \& Allis, 2001). The histone code influences higher-order chromatin structures by affecting contacts between different histones, and between the histones and the DNA. Specific histone modifications are responsible for the compartmentalization of the genome into distinct domains, such as transcriptionally silent heterochromatin and transcriptionally active euchromatin (summarized by Martin \& Zhang, 2005). The recent term 'heterochromatin' is more loosely applied; it is often extended to include transcriptionally silent regions of the chromatin, regardless of their staining properties. An important distinction is made between the constitutive and facultative heterochromatin. Constitutive heterochromatin is always compact, and tends to be enriched in repetitive, gene-poor, and late replicating DNA sequences; whereas, facultative heterochromatin can undergo reversible transitions from a compact, transcriptionally inactive state to become more open and transcriptionally competent. In a review, Trojer and Reinberg (2007) suggested that facultative heterochromatin should be molecularly defined as condensed, transcriptionally silent chromatin regions that decondense, and allow for transcription within temporal, spatial, or parental/heritable contexts. For example, during embryogenesis, the amount of facultative heterochromatin increases, as unwanted sets of genes are progressively shut down until maturity, when a cell expresses only the genes appropriate for that tissue. The reverse occurs when, for example, differentiated cells are reprogrammed to become stem cells. These events are typically accompanied by profound changes in histone variants, histone modifications, and the presence of chromatin attaching proteins (reviewed in Woodcock \& Ghosh, 2010). The ability of the histone code to dictate the chromatin environment allows it to regulate nuclear processes, such as replication, transcription, DNA repair, and chromosome condensation (Kouzarides, 2007).

Next to DNA methylation, histone acetylation and histone methylation are the bestcharacterized epigenetic markers. Trimethylation at H3K4, H3K36, or H3K79 results in an open chromatin configuration, and therefore is characteristic of euchromatin. Euchromatin 
is also characterized by a high level of histone acetylation, which is mediated by histone acetyl transferases (HATs). Conversely, histone deacetylases (HDACs) have the ability to remove this epigenetic mark, which leads to transcriptional repression. Condensed heterochromatin is enriched in the trimethylation of H3K9, K3K27, and H4K20 (Kouzarides, 2007), which along with the silencing of euchromatin loci, caused by histone deacetylation, involves the recruitment of specific K9 histone methyltransferases (HMTs). Methylated H3K9 provides a binding site for the chromodomain-containing heterochromatin protein 1 (HP1), which induces transcriptional repression and heterochromatinization. At euchromatic loci, this process is mediated by co-repressors, such as retinoblastoma protein pRb or KAP1 (summarized by Kouzarides, 2007). Histone demethylases, first described by Shi et al. (2004), have the opposite effect on transcription. The histone demethylase LSD1 is responsible for $\mathrm{H} 3 \mathrm{~K} 4$ demethylation, which leads to transcriptional inactivation. (reviewed in Bartova et al., 2008).

In contrast to the large amounts of available information on the functions of HATs, HDACs, and enzymes that mediate histone methylation (listed in Kouzarides, 2007), enzymes responsible for other types of histone modifications, such as phosphorylation and ubiquitination, are not as intensively studied (Grant, 2001, as cited in Bartova et al., 2008). This is despite the fact that these modifications have important roles in transcription, DNA repair, the induction of apoptosis, and chromosome condensation (Cheung et al., 2000, as cited in Bartova et al., 2008). For example, the phosphorylation of serine 10 in histone $\mathrm{H} 3$ is associated with transcriptional activation in mammalian cells (Thomson et al., 1999, as cited in Bartova et al., 2008); and H2A phosphorylation is responsible for chromosome condensation (Grant, 2001, as cited in Bartova et al., 2008). These observations underscore the importance and complexity of histone modifications in the regulation of nuclear, and subsequently, cellular processes (Bartova et al., 2008).

\subsection{DNA repair in heterochromatin and euchromatin}

Phosphorylated histone $\mathrm{H} 2 \mathrm{AX}(\mathrm{\gamma H} 2 \mathrm{AX})$ are widely used as a specific marker of DSBs, and the immunostaining of $\gamma \mathrm{H} 2 \mathrm{AX}$ foci allows for the most sensitive monitoring of DSB induction (with ionizing radiation doses in the order of cGy or even mGy), with the cell nucleus and also of DSB repair. One striking observation about $\gamma \mathrm{H} 2 \mathrm{AX}$ is that foci are more rarely detected at heterochromatic sites than in the euchromatin. $\gamma \mathrm{H} 2 \mathrm{AX}$ foci distribution within irradiated cells is uneven, as foci can only be detected at the periphery of heterochromatic regions, rather than within them; the boundaries of which are maintained by methylation of lysine at position 9 on histone $\mathrm{H} 3$ (H3K9), an important epigenomic imprint of heterochromatic regions. However, there is still debate as to the significance of this resistance of heterochromatin to $\mathrm{gH} 2 \mathrm{AX}$ foci formation. The $\mathrm{H} 2 \mathrm{AX}$ in the heterochromatin may simply be inaccessible to phosphorylation because of the compact nature of the chromatin; or in fact, the heterochromatin might be more resistant to DNA damage (Cowell et al., 2007; Falk et al., 2010; reviewed in Cann \& Dellaire, 2011). Falk et al. (2008) used an immunoFISH method (immunocytochemistry combined with fluorescence in situ hybridization) to analyse DSB formation in chromosomes as well as in chromatin regions with known chromatin compaction. They found that condensed regions of chromatin (with a low density of expressed genes) known as anti-Ridges, and also interphase domains of gene-poor and transcriptionally less active chromosomes were less susceptible to DSB induction, compared with decondensed chromosomes and decondensed 
gene-rich chromatin; with the caveat that gH2AX was still being used as the DSB marker (Falk et al., 2008). They speculated that the high amount of protein bound to the heterochromatin, including HP1, acts as a protective layer that prevents access to the DNA by ROS (Falk et al., 2008). Indeed, approximately 56\% of g-irradiation-induced DSBs are indirectly caused by hydroxyl radicals, rather than directly by the g-irradiation itself. As such, the decondensed and hydrated euchromatin would be much more susceptible to this secondary damage (reviewed in Falk et al., 2010). It is easy to see how this model of heterochromatin protein-based protection could be extrapolated to other DNA-damaging agents, such as UV irradiation and mutagenic chemicals. Furthermore, increasing the accessibility of chromatin to DNA-damaging agents is one of the proposed mechanisms of action of histone deacetylase (HDAC) inhibitors, with respect to their ability to sensitize cells to chemotherapeutic agents (Kim et al., 2003, as cited in Cann \& Dellaire, 2011).

An additional confounding observation is that following DSBs, chromatin rapidly decondenses in the vicinity of the break (see below Kruhlak et al., 2006; Falk et al., 2007; Dellaire et al., 2009) and undergoes histone acetylation (Falk et al., 2007). Goodarzi et al. (2008) proposed a model for heterochromatic DSBs, which are generally repaired more slowly than are euchromatic ones. This model involves ATM signalling, required for DSB repair in heterochromatin, which affect $<25 \%$ of the DSBs. The ATM protein belongs to a growing family of PIKK kinases, which is observed at the sites of DNA damage, and where it is autophosphorylated and dissociated from its nonactive dimeric form into the active monomeric form. Direct connections between chromatin alteration and ATM were made when the transcriptional corepressor Krüppel-associated box (KRAB)-associated protein (KAP)-1 was identified as an ATM substrate, being robustly phosphorylated at S824; causing transient chromatin relaxation (Ziv et al., 2006, as cited in Cann \& Dellaire, 2011). KAP-1 (also called TIF1b, TRIM28, or KRIP-1) is an abundant nuclear protein that binds to KRAB domains within sequence-specific transcriptional repressors to trigger heterochromatin formation via interactions with proteins, such as heterochromatin protein 1 (HP1), HDACs, SET-domain histone methyltransferases, and ATP-dependent chromatin remodelers (Craig, 2005, as cited in Goodarzi et al., 2008). Nevertheless, exactly how KAP1 mediates this decondensation is still unknown, as its mobility, its association with chromatin, and its interaction with its heterochromatic mediators are relatively unchanged following phosphorylation (reviewed by Cann \& Dellaire, 2011).

In the absence of ATM signalling, heterochromatic repair stalls and the lesion persists. Significantly, the knockdown of the transcriptional repressor KAP-1, an ATM substrate, or of the heterochromatin-building factors $\mathrm{HP} 1$ or $\mathrm{HDAC} 1 / 2$, alleviates the requirement for ATM in DSB repair. Moreover, cells exhibiting heterochromatin disorganization caused by mutations in the DNA or histone methyltransferases do not require ATM for heterochromatic DSB repair. The requirement of ATM in DNA repair is also affected by lamina disruption, as the result of LMNA gene mutations. Lamins play a significant role in heterochromatin; moreover, in the interphase chromosome organization at the nuclear periphery (Ondrej et al., 2008). Interestingly, transient induction of chromatin hypercondensation by hyperosmotic treatment of the cells led to the formation of a small dot of $\gamma \mathrm{H} 2 \mathrm{AX}$, dispersion of ATM, and its additional partners in the signalling pathways (53BP, NBS1) through the cell nucleus. The signals of $\gamma \mathrm{H} 2 \mathrm{AX}$ and ATM or 53BP did not overlap. Cell transfer into isotonic medium for the subsequent 10 minutes led to the complete restoration of chromatin structure, as well as the formation of $\gamma \mathrm{H} 2 \mathrm{AX}$ foci and the assembly of ATM and 53BP at the sites of DNA damage (Falk et al., 2008). 
In euchromatin, ATM signalling perturbs the local chromatin architecture which, while important for optimal signalling, is dispensable for repair, since NHEJ factors can freely access or manipulate the DSB. Hence, $>75 \%$ of DSBs in G0/G1 are repaired in an ATM-independent manner. Interestingly, lower eukaryotes, which have minimal heterochromatin, have a diminished role for ATM signalling in their DSB response (Morrow et al., 1995, as cited in Goodarzi et al., 2008). By contrast, ATM signalling is of major importance to the higher eukaryotic DSB response (Lavin et al., 2005, as cited in Goodarzi et al., 2008), where a larger genome and complex developmental program resulted in more constitutive and facultative heterochromatin. It suggests that the increased prominence of ATM signalling in the DSB response (compared with TEL1 signalling in yeast) correlates with the increasing complexity of chromatin architecture observed throughout eukaryotic evolution (Goodarzi et al., 2008).

As introduced above, HP1 is important in heterochromatin formation. It can bind H3K9me3, as well as the DNA methyltransferases DNMT1 and DNMT3a, which are involved in CpG island methylation, and the histone methyltransferase Suv39H1 (reviewed in Cann \& Dellaire, 2011). Given that Suv39H1 can trimethylate H3K9, and that HP1 can interact with both H3K9 and Suv39H1, HP1 function has been proposed as a mechanism for heterochromatin expansion (Maison \& Almouzni, 2004, as cited in Cann \& Dellaire, 2011). Mammalian cells have 3 HP1 isoforms: HP1a, HP1b, and HP1g. These proteins can form homo- and heterodimers through their C-terminal chromoshadow domains; with this selfassociation potentially helping to compact the chromatin (Maison \& Almouzni, 2004, as cited in Cann \& Dellaire, 2011). The dimerization of 2 chromoshadow domains also creates a hydrophobic surface that can bind proteins such as KAP1 (Lechner et al., 2005, as cited in Cann \& Dellaire, 2011). While the HP1 proteins localize to, and are involved in, the assembly and maintenance of heterochromatin at the centromeres and telomeres, they are also involved in the regulation of transcription in both euchromatin and heterochromatin (Dinant \& Luijsterburg, 2009). Goodarzi et al. (2008) demonstrated that depletion of all three HP1 isoforms removed the requirement for ATM in the repair of heterochromatic DSBs, suggesting that HP1-mediated chromatin compaction is inhibitory to DNA repair. Indeed, HP1b exhibits a transient release from damaged sites, with casein kinase 2-mediated phosphorylation of Thr51 being the proposed initiator of release (Ayoub et al. 2009b). This residue is within the chromodomain of HP1, which is responsible for its ability to bind H3K9me3 (Ayoub et al., 2009b). However, all three HP1 isoforms have also been shown to be recruited to sites of DNA damage, including UV-induced cyclobutane pyrimidine dimmers and pyrimidine(6-4)pyrimidone photoproducts (Luijsterburg et al., 2009), oxidative lesions (Zarebski et al., 2009), and DNA DSBs (Ayoub et al., 2008, 2009a; Luijsterburg et al., 2009, reviewed in Cann \& Dellaire, 2011). The recruitment of HP1 to sites of DSBs was found to be dependent on the chromoshadow domain and independent of the chromodomain and H3K9me3 (Luijsterburg et al., 2009). In an attempt to rationalize these conflicting results, a bimodal model for HP1 dynamics following DSBs has been proposed, with an initial phosphorylation-dependent release of HP1, and a subsequent accumulation of HP1 at sites of DNA damage (Ayoub et al., 2009b; Dinant \& Luijsterburg, 2009; Zarebski et al., 2009). In this model (reviewed in Cann \& Dellaire, 2011), the initial release of HP1 from H3K9me3 would help effect chromatin decondensation. The subsequent chromoshadow dependent accumulation of HP1 might then help mediate and enhance the DNA damage signalling. Indeed, HP1 has also been shown to be recruited to sites of heterochromatic perturbation (Zhang et al. 2007), suggesting that it could help stabilize 
damaged chromatin. Furthermore, phosphorylated HP1 might provide an epitope for mediators of the DNA damage response pathway, many of which bind phosphorylated proteins (Dinant \& Luijsterburg, 2009).

At present, a lot is known about the biochemical aspects of DSB repair, but very little about how chromatin structure influences this process and the sensitivity of DNA to DSB induction. We lack the answers even to the very basic questions formulated by Falk et al. (2010) such as: Are DSBs induced equally in functionally and structurally distinct chromatin domains? Where and how are DSBs actually repaired: individually at their sites of origin, or together in specialized, repair-competent nuclear sub-compartments. Are DSBs spatially stable or do they migrate into "repair factories". Further, what are the DSB clusters observed by several authors: (Kruhlak et al., 2006; Falk et al., 2007) sites of multiple DNA damage, repair factories, or by-products of DSB repair that potentially increase the risk of chromatin mis-rejoining? Consequently, which is the more important factor that underlies chromosomal translocations - the predetermined nuclear proximity of DSBs ("position first" hypothesis) in the range of Brownian movement of chromatin (about $0.5 \mathrm{~mm}$ ), or in their more extensive, potentially directed movement during the repair process that brings them close to one another (the "breakage first" hypothesis)?

\subsection{Chromatin loops - Structure, organization and mobility}

Chromatin loops are an ubiquitous structural element of chromatin, which is important in many scenarios of chromatin function and dynamics (van Driel et al., 2003; Fraser, 2006). They are attractive organizational and regulatory features, because they provide structural support to the chromatin fibre; at the same time, they bring distantly located sequence elements into spatial proximity, allowing for regulatory communication between these sites. Vice-versa, loops can spatially segregate genomic regions from one another, and ensure their independent function. Loops have been implicated at virtually all levels of chromatin organization, and they function ranging from kilobase-sized loops involved in the interaction of upstream elements with promoters, to giant loops of hundreds of kilobases which might contribute to gene placement away from the chromosome body and into distinct nuclear environments (van Driel et al., 2003; Cremer et al., 2006; Misteli, 2007). The existence and physiological relevance of the various types of loops at times is difficult to ascertain, as they often cannot be detected under native conditions and are generally refractory to visualization in situ. Regardless, the relevance of loops in several generegulatory events has recently been reinforced (Fraser, 2006; reviewed in Misteli, 2007). In the past, the concept of chromatin loops involved an insoluble proteinaceous nuclear matrix or nucleoskeleton (to which chromatin loops are anchored by DNA sequences referred to as matrix attachment regions (MARs)). However, because it has not been possible to define its composition and structure, the matrix remains a useful working concept, rather than a wellaccepted structure in the same sense as the cytoskeleton (Pederson, 2000). Nonetheless, recent findings concerning lamins and nuclear actin could revive the idea of a nucleoskeleton; however, not as the main foundation of chromatin organization.

To address the topology and organization of the loops, several authors have measured the two-dimensional spatial distance between the genomic markers as a function of their genomic separation (distance). Two different models have been presented. The first is called the Random-Walk/Giant-Loop (RW/GL) Interphase Model. In this model, below $\sim 2 \mathrm{Mbp}$, the chromatin fibre seems to rapidly extend with the genomic separation; and above $\sim 2 \mathrm{Mbp}$ a random walk behaviour was found. Therefore, Sachs et al. (1995) assumed loops of 1 to 5 
Mbp, which are attached to a non-DNA (and presumably protein) backbone, which is in agreement with the nuclear matrix hypothesis (although not yet supported). Fitting the experimental distance measurements (Yokota et al., 1995) with this extended analytical model revealed a loop size of $\sim 3 \mathrm{Mbp}$, and separations of the attachment points of the giant loops of $620 \mathrm{~nm}$. More accurate spatial distance measurements between the genetic markers, as a function of their genomic separation using a more structure preserving protocol, also showed biphasic behaviours. However, the increase in the distance was slower with growing separations below $\sim 2 \mathrm{Mbp}$; implying much smaller loops than in the RW/GL model. Thus, the smaller loops need to be aggregated into connected clusters, which for larger genetic separations are arranged like a random walk. A detailed interpretation of these findings resulted in the proposal of a Multi-Loop-Subcompartment (MLS) interphase-metaphase model (Múnkel et al., 1998). In this model, small loops of 60 - $120 \mathrm{kbp}$ from rosettes in the interphase, are linked by chromatin linkers (again of $\sim 120 \mathrm{kbp}$ ). This is also in agreement with the general dynamics of chromatin, showing a short-range motion that is locally constrained within random directions. Nevertheless, as mentioned above, Goetze et al. (2006) showed that the compaction of chromatin is linked to the size of the loops, and distinguishes chromatin from compact heterochromatin and decondensed euchromatin. Except for short-range motion, the long-range motion of the chromatin has also been observed within the nucleus. Some chromatin regions occasionally showed large $(\sim 0.5 \mu \mathrm{m})$ directional movements, over timeperiods as short as 10 seconds, which were inhibited in ATP-depleted cells. The large-range motion of the loops (giant loops) is connected with the transcription of the genes, located in that chromatin region. Dundr et al. (2007) showed that the movement of synthetic inducible U2 snRNA gene arrays over long distances inside the nucleus $(2-3 \mu \mathrm{m})$ became associated with the Cajal bodies. Interestingly, the expression of these arrays and their movement was dependent on the ability of nuclear actin to polymerize. These findings showed that some parts of the chromatin could undergo changes via the RW/GL model.

As described above, chromatin has the potential for large-range motion in some cases; however, we do not know if DSBs are similarly able to move. That DSBs can form clusters has been described. For example, Markova et al. (2007) correspondingly quantified the area of $\mathrm{gH} 2 \mathrm{AX} / 53 \mathrm{BP} 1$ foci induced by g-radiation during the post-irradiation period, and surprisingly found that a progressive decrease in the number of gH2AX/53BP1 foci was not followed by an equivalent decrease in the overall area of foci; instead, it remained unchanged, up to $4 \mathrm{~h}$ post irradiation. However, if these clusters are the results of longrange motion of the chromatin is still not clear.

\subsection{DSB mobility and clustering}

Chromatin decondensation at the break sites is probably connected with frequent DSB protruding into "chromatin holes"; an area of low chromatin density from boundary regions, with areas of high chromatin density (Falk et al., 2007). It has been demonstrated that most gH2AX foci induced by g-radiation are spatially stable; however, some "breaks" show a significantly higher mobility. When the mobility was quantified as the mean change of the distances between all possible DSB pairs over time (53BP1 or NBS1 foci), the mobility of the damaged chromatin was equivalent to that of the undamaged. However, when tracking individual foci, a subgroup with significantly increased mobility was identified. To explain this, Falk et al. (2007) double-transfected MCF7 cells with the 53BP1 protein, and $\mathrm{H} 2 \mathrm{~B}$ histone fused with GFP and RFP, respectively (so that one could follow the movement of 53BP1 foci relative to chromatin density). Interestingly, the foci showing increased (but 
restricted) movement frequently corresponded with heterochromatic DSBs. This movement, although seemingly random when studied at the pan-nuclear level, was from high-density chromatin at the sites of DSB origin, to immediately adjacent domains of sparse chromatin.
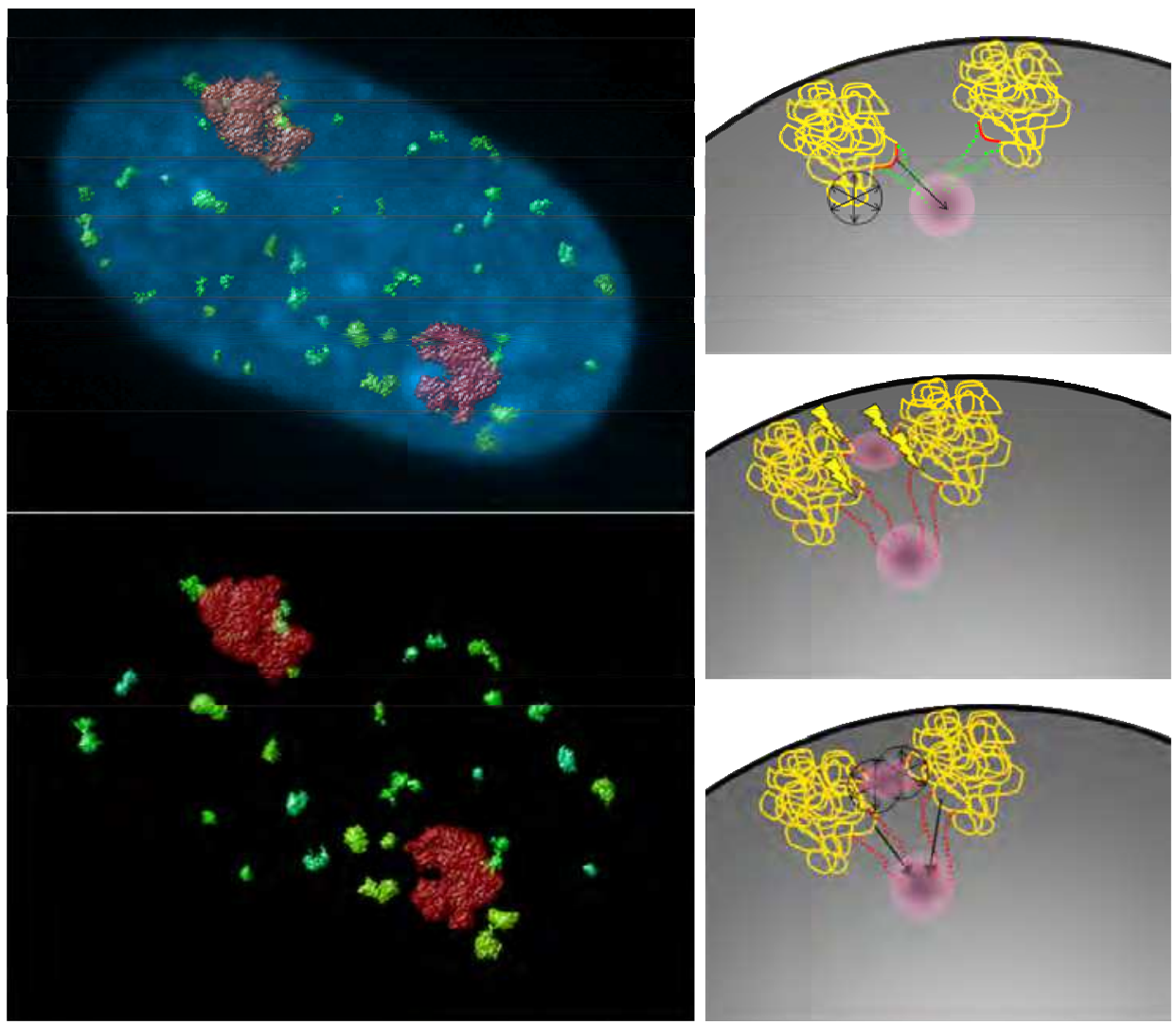

Fig. 1. A deconvolved image of the human fibroblast nucleus, irradiated with a dose of 2 Gy. $\gamma \mathrm{H} 2 \mathrm{AX}$ foci represented DSBs are dispersed within the nucleus, mostly out of heterochromatin (chromatin is stained by DAPI - blue). Some of $\gamma \mathrm{H} 2 \mathrm{AX}$ foci are in close proximity to form signals such as double-dots. CT of HSA1 (red) is visualized by the FISH technique. $\gamma \mathrm{H} 2 \mathrm{AX}$ foci are located on the surface of the chromatin mass of the CTs, not inside. A schematic drawing of chromatin mobility. The upper subset figure shows the possibilities of chromatin motion - constrained random short-range motion (black circle with arrows) and directional long-range motion (arrows) during transcriptional activation of a given region (red - inactive changes to active - green interrupted line). The active genes are shifted to the transcription factory (pink circle). The middle figure of the subset demonstrates clustering of the damaged chromatin loci (red interrupted lines). The pink circles represent only the location of random clustering or hypothetical repair factories. The lower figure of the subset shows the hypothetical possibility of DSBs' motion to form the clusters or repair factories (pink circles) - constrained random short-range motion (black circle with arrows) and active directional long-range motion (arrows) 
As reviewed by Falk et al. (2010), DSB clusters are formed at sites that differ from the original positions of DSB lesions. At first, it was thought that the presence of these clusters confirmed the existence of the "repair factories" described above. However, contrary to the frequent clustering of foci in cells irradiated with high-LET radiation, only a few clusters per cell (each usually containing only 2 or $3 \mathrm{gH} 2 \mathrm{AX}$ foci) appear after a dose of $1 \mathrm{~Gy}(1 \mathrm{~Gy} / \mathrm{min})$ of g-rays (Falk et al., 2007). This means that no more than $5-8$ gH2AX foci out of about 35 DSBs induced per cell (on average) after this dosage usually form clusters; and most foci have not entered clusters, even several hours after g-irradiation. These results do not support the hypothesis of "repair factories", and the clustering of gH2AX foci after airradiation apparently reflects only the random "movement" of chromatin. Moreover, soon after irradiation (5 min. post irradiation), the frequency of clustered foci was low, but increased with time post irradiation, and DSB clustering occurs preferentially in chromatin holes, regions with very low chromatin concentration (Falk et al., 2007). Falk and co-workers (2010) also proposed that clusters represent unrepaired DSBs, since they still co-localized with other repair proteins (MRE11, NBS1, and 53BP1). It indicates that DSBs in clusters could only be repaired with difficulty; it is therefore tempting to speculate that they represent sites with an increased risk of chromosomal aberrations. Indeed, Scherthan et al. (2008, as cited in Falk et al., 2010) recently reported results that strongly support this hypothesis. Comparing the numbers of gH2AX foci induced by different doses of X-rays with corresponding frequencies of chromosomal aberrations detected by spectral karyotyping (SKY), they showed that more DSBs accumulate in a single gH2AX focus after a higher dose of IR; this correlates with an increased risk of DNA mis-rejoining, and the formation of complex aberrations. In addition, it seems that clustered foci also contribute to the process of cell adaptation to ionizing radiation, as reviewed in Falk et al., 2010. This process enhances the risk of fixing chromosomal aberrations, because it allows the cell to enter mitosis with unrepaired DSB damage (reviewed in Falk et al., 2010).

\section{Chromosome territories in theories of chromosomal translocation origin}

To answer questions concerning chromosomal aberrations and the hypothesis of their origin (the "position first" and "breakage first" hypotheses), it is necessary to clearly understand the spatial organization of the genome. The most global level of cellular genome organization is the arrangement of the genome regions within the $3 \mathrm{D}$ space of the cell nucleus (Cremer et al., 2006; Meaburn \& Misteli, 2007; Misteli, 2007). The nonrandom nature of spatial genome organization is indicated by the age-old observation of the segregation of transcriptionally active and inactive regions into physically separate domains of euchromatin and heterochromatin, respectively. Recently, more-detailed mapping studies of smaller genome regions have significantly extended this concept and have made it clear that chromosomes, genome regions, and single genes are nonrandomly arranged within the nucleus (Cremer et al., 2006). Changes in positioning patterns occur during differentiation and development; strongly suggesting a link between the positioning and genome function (Parada et al., 2004; Cremer et al., 2006; reviewed in Misteli, 2007).

\subsection{CT theory, structure, organizations, and models}

In the interphase nuclei, decondensed mitotic chromosomes exist as chromosome territories. They are defined as the nuclear space taken up by the DNA of a given chromosome; the 
term "territory" refers to the fact that the occupied nuclear space is compact, typically roughly in shape, and with a volume of about $2-3 \mu \mathrm{m}$ in diameter (reviewed in Cremer \& Cremer, 2001; Misteli, 2007; Misteli, 2010). Chromosome territories (CTs) represent the highest order of chromatin structure and the largest unit of organization of the eukaryotic genome. Speculation about a chromosome territory concept has developed since the late $19^{\text {th }}$ century. The territorial organization of interphase chromosomes was first suggested for animal cell nuclei by Carl Rabl (Rabl, 1885, as cited in Cremer \& Cremer, 2010), but it was Theodor Boveri who introduced the term chromosome territory in his seminal studies of blastomere stages of the horse roundworm Parascaris equorum or Ascaris megalocephala (Cremer \& Cremer, 2010). However, the direct visualization of individual CTs was made possible by in situ hybridization techniques developed during the mid-1980s. The achievement of chromosome sorting by flow cytometry of fluorescently-labelled mitotic chromosomes (Cremer et al., 1984a; Gray et al., 1987; Fawcett et al., 1994, all as cited in Cremer \& Cremer, 2010) enabled the generation of chromosome specific painting probes for a large number of species. Subsequent amplification of DNA by cloning in bacterial vectors; or by universal PCR (Telenius et al., 1992, as cited in Cremer \& Cremer, 2010), as well as novel techniques for the suppression of ubiquitous repetitive sequences by COT-1 DNA (Cremer et al., 1988; Pinkel et al., 1988; Lichter et al., 1988a; Lichter et al., 1988b, all as cited in Cremer \& Cremer, 2010); likewise, the depletion of these sequences from the respective probes (Bolzer et al., 1999, as cited in Cremer \& Cremer, 2010), made it possible to delineate individual chromosomes in metaphase plates and their territories in the interphase nucleus (reviewed in Cremer \& Cremer, 2010).

In the conventional model of mammalian nuclear architecture, the arrangement of the chromosome territories within the interphase nucleus is nonrandom in two ways - radial distribution and nonrandom neighborhood arrangements. Radial distribution is characterized by gene-rich chromosome territories' occupation of the interior regions of the interphase nucleus; whereas, gene-poor domains are localized to the nuclear periphery. This general arrangement was confirmed by analyses comprising all human chromosomes (Boyle et al., 2001). An evolutionary comparison of lymphoblastoid cells from various primate species showed that this nonrandom radial nuclear distribution has been evolutionarily conserved despite major evolutionary chromosome rearrangements (reviewed in Cremer \& Cremer, 2010). Nonrandom radial nuclear arrangements of CTs, depending on their gene density, were also found in other mammals like rodents (Mayer et al., 2005; Neusser et al., 2007, all as cited in Cremer \& Cremer, 2010) and cattle (Koehler et al., 2009, as cited in Cremer \& Cremer, 2010), additionally in birds (Habermann et al., 2001, as cited in Cremer \& Cremer, 2010). Recently, it was shown in bovine pre-implantation embryos that this difference was not yet present in the nuclei of early blastomere stages. Its first appearance in correlation with major genome activation, and was fully established in blastocysts (Koehler et al., 2009; as cited in Cremer \& Cremer, 2010).

CTs have preferred positions, not only with respect to the centre or periphery of the nucleus, but also with respect to one another (Parada \& Misteli, 2002). This circumstance of the nonrandom arrangement is referred to as proximity patterns. However, these proximity patterns were of a rather probabilistic nature, i.e., their presence was shown by an excess of certain heterologous (and occasionally also homologous) CTs in a population of nuclei, not as an event consistently observed in each nucleus (Cremer \& Cremer, 2010). Differences of statistically preferred proximity patterns varies between cell types, and it has consequences 
for a chromosome's ability to interact in trans with other parts of the genome, as revealed by the frequency of specific chromosome translocations (reviewed in Fraser \& Bickmore, 2007). The nonrandom organization of the genome in 3D space of the nucleus is explained by selforganization of the chromatin (reviewed in Misteli, 2010). Although the nuclear model with protein matrix was rejected, recent studies increased the importance of a peripheral protein network based on lamins - the nuclear lamina (NL). Microscopy studies have indicated that specific regions of the chromosomes are located in close proximity to the NL, and that the chromatin is specifically attached to the NL. It has also been demonstrated that the human genome contains more than 1,300 sharply defined large domains 0.1 - 10 megabases in size, characterized by genome-lamina interactions. These lamina-associated domains (LADs) are typified by low gene-expression levels, indicating that LADs represent a repressive chromatin environment. Taken together, it demonstrates that the human genome is divided into large, discrete domains that are units of chromosome organization within the nucleus (Guelen et al., 2008).

CTs can be further classified into sub-chromosomal domains such as chromosome arms, centromeres, or telomeres. It has not only been demonstrated that CTs as a whole, but also sub-domains like the chromosome arms occupy discrete areas in the nuclear volume (Dietzel et al., 1998). Centromeres and telomeres, whose main functional role is to maintain the chromosome integrity, also do locate in distinct sub-volumes shown in various FISH experiments with specific probes (Molenaar et al., 2003; Weierich et al., 2003). In addition, sub-chromosomal domains undergo peripheral genome positioning, depending on genedensity and transcriptional activity of a given domain. For instance, $\mathrm{R}$ bands of chromosomes are generally found toward the centre of the nucleus, whereas latereplicating, inactive $\mathrm{G}$ bands are often located toward the periphery (Ferreira et al., 1997; Sadoni et al., 1999; Gilbert et al., 2004). Goetze et al. (2007) found that ridges (gene rich domains containing both housekeeping genes and highly expressed genes) and anti-ridges (relative gene poor domains with low level of gene expression) of chromosomes 1 and 11 occupy different radial positions. The two ridges are predominantly located more towards the nuclear interior than the three anti-ridges, which on average are closer to the nuclear envelope.

Several groups have documented the relationship between gene topology within the CT and its transcriptional activity (Dietzel et al., 1999; Bartova et al., 2002). Active gene loci were found on the surface of the chromatin mass of CT, and the same inactive genes were positioned towards the territory's interior (reviewed in Cremer \& Cremer, 2001). Moreover, a few chromatin regions with particularly high gene density and/or transcriptional activity, such as the 11p15.5 segment, the MHC and EDC loci, or the HOX gene cluster have been consistently found looping out as protrusions from the core territory (Volpi et al., 2000; Williams et al., 2002; Chambeyron et al., 2005; Kupper et al., 2007).

All of the above-mentioned findings led to the creation of models of the functional architecture of CTs and the nucleus. The chromosome territory-interchromatin compartment (CT-IC) model proposes that nuclei are built up from two principal components: chromosome territories, and the interchromatin compartment (IC). The IC represents a The DNA-free (or at least largely free) contiguous space of the channels, starts at the nuclear pores and expands as larger channels and lacunas between the higher-order chromatin network, described above. The IC harbours splicing speckles and a variety of nonchromatin nuclear bodies (Verschure et al., 1999; Visser et al., 2000; Albiez et al., 2006). The IC concept 

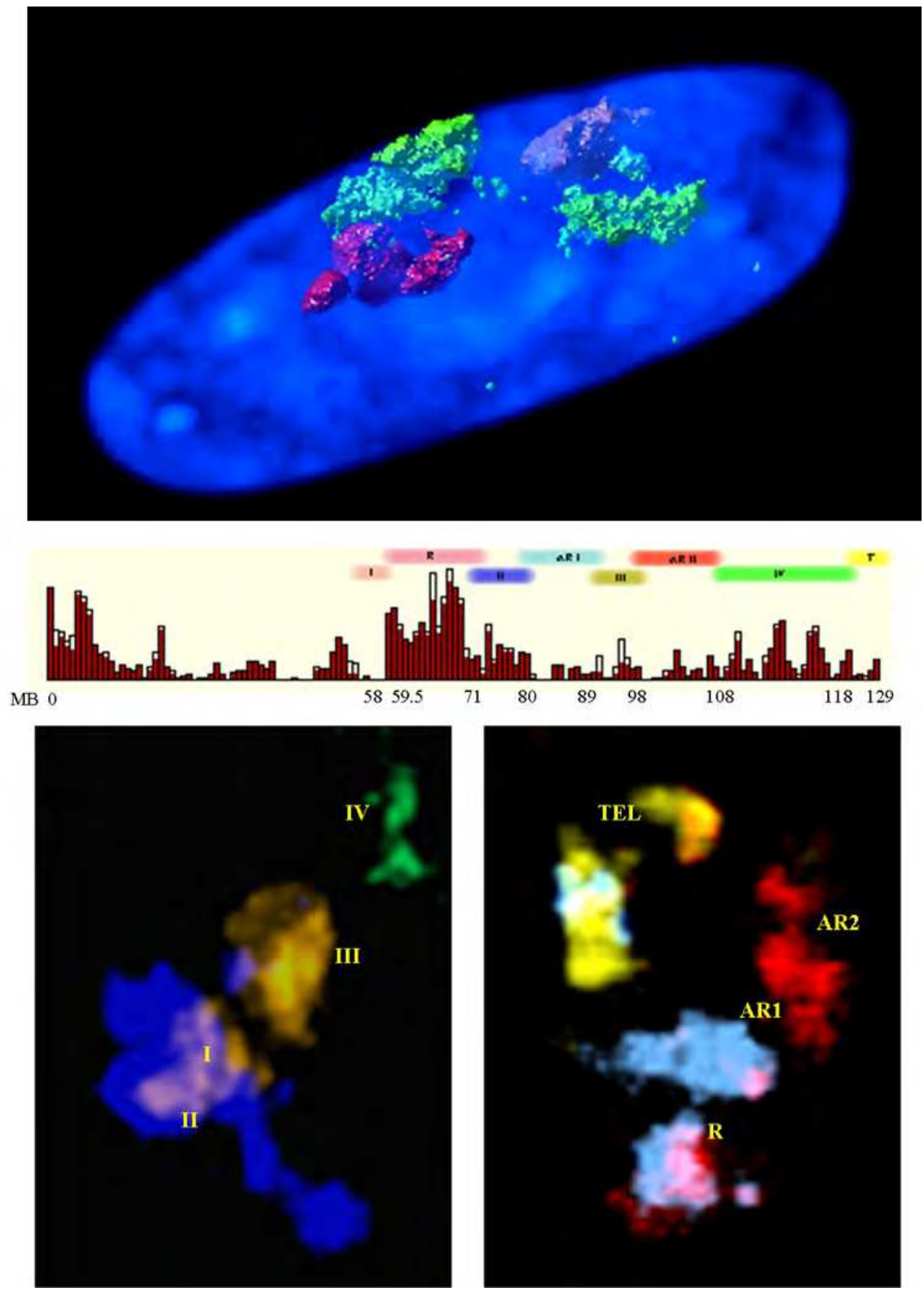

Fig. 2. A deconvolved image of chromosome territories of HAS 1 (green) and 2 (red) within the nucleus of a human fibroblast. Multicolor FISH of specific subdomains of chromosome territory HAS 11, showing radial arrangements of the subdomains in relationship to gene expression activity. Highly transcriptionally active domains ( $\mathrm{R}$ - ridges, and domains marked I and II) are oriented to the nuclear centre, less active domains (AR1, AR2 - antiridges, domains marked as III, IV, and the telomeric region) are located more peripherally. 
evolved from the interchromosomal domain (ICD) concept originally proposed by Zirbel et al. (1993, as cited in Cremer \& Cremer, 2010), who defined the ICD as a network-like space mainly expanding around CTs, with little penetration into the CT interior (Cremer et al., 1993, as cited in Cremer \& Cremer, 2010). Supposedly, genes were preferentially transcribed in a region of decondensed chromatin at the CT periphery, and RNA transcripts would be directly released into the ICD compartment (reviewed in Cremer \& Cremer, 2010). However, a physical separation between CTs is not supported by the data on translocation frequencies and chromatin dynamics. Simulations of chromosome translocations, based on models of chromosome organization, have suggested the existence of a significant degree of intermingling between CTs (Hlatky et al., 2002; Holley et al., 2002; Sachs et al., 2000, all as cited in Branco \& Pombo, 2006).

Using the novel FISH procedure for ultrathin cryosections (approximately $150 \mathrm{~nm}$ thick; cryo-FISH) of well-fixed, sucrose-embedded cells, which maximizes chromosome-painting efficiency, provides high resolution; and it simultaneously preserves chromatin nanostructure (Branco \& Pombo, 2006). It has been shown that chromosomes intermingle significantly in the interphase nuclei of human cells, and depending on CT compaction, as well; this argues against the presence of an interchromosomal domain that separates CTs. The extent with which particular pairs of CTs intermingle correlates with the frequency of chromosome translocations in the same cell type (Branco \& Pombo, 2006). According to these results, the interchromatin network (ICN) model was proposed (Branco \& Pombo, 2006). This model predicts that chromatin fibres and loops intermingle in a rather uniform fashion, both in the interior of individual CTs and between differentially labelled neighbouring CTs; making any distinction between the interior or periphery of distinct chromatin domains functionally meaningless (reviewed in Cremer \& Cremer, 2010). Albiez et al. (2006) undertook to resolve these conflicting views. Using experimental manipulations of the nuclear architecture, these authors obtained results supporting the CT-IC model, but they did not confirm the conclusions of the ICN model. On the other hand, a certain degree of intermingling between neighbouring territories and their physical distance may be important for the occurrence of chromosome translocations. Thus, IC represents space where chromatin loops of a different $\mathrm{CT}$, with genes and regulatory elements, could meet, co-localize, and regulate themselves; furthermore, they could also create a transcription factory (Fraser \& Bickmore, 2007).

\subsection{Spatial CT organization in cancer}

The organization of chromosome territories has functional consequences for genome organization and its maintenance; but also for the regulation of gene expression (reviewed in Cremer \& Cremer, 2010). It is therefore not surprising that defects in higher-order chromatin structures and chromosome organization, such as translocations, cause diseases. Chromosomal abnormalities in the form of translocations are a general hallmark of cancer cells. The translocations occur during interphase by illegitimate joining of two or multiple chromosomes containing persistently damaged DNA such as DSBs requiring physical interaction of them. Thus, it is not surprising that the 3D proximity, CT volume, and the intermingling volume of CTs increases the translocation frequency between them (Branco \& Pombo, 2006). This is a particularly intriguing and important finding in light of the nonrandomness in spatial genome organization, enabling determination of potential translocation partners (reviewed in Meaburn et al., 2007; Misteli, 2010). The contribution of 
spatial proximity to translocation formation is illustrated by the frequent formation of Robertsonian translocations, arising by the fusion of two acrocentric chromosomes, which in humans always contains nucleolar organizing regions (NORs), which cluster together in the nucleoli. Furthermore, translocations amongst peripheral chromosomes such as HSA4, 9, 13, and 18 occur at higher frequencies than with internally localized chromosomes, presumably due to their closer physical localization to each other (reviewed in Maeburn et al., 2007). Furthermore, when cells which contain nonrandom pairs of closely positioned chromosome 2 and 5 were irradiated to induce DSBs, these two chromosomes formed translocations with a significantly higher frequency than in cells where they were distant from one another; suggesting that their proximity facilitated their translocation (Mathas et al., 2009).
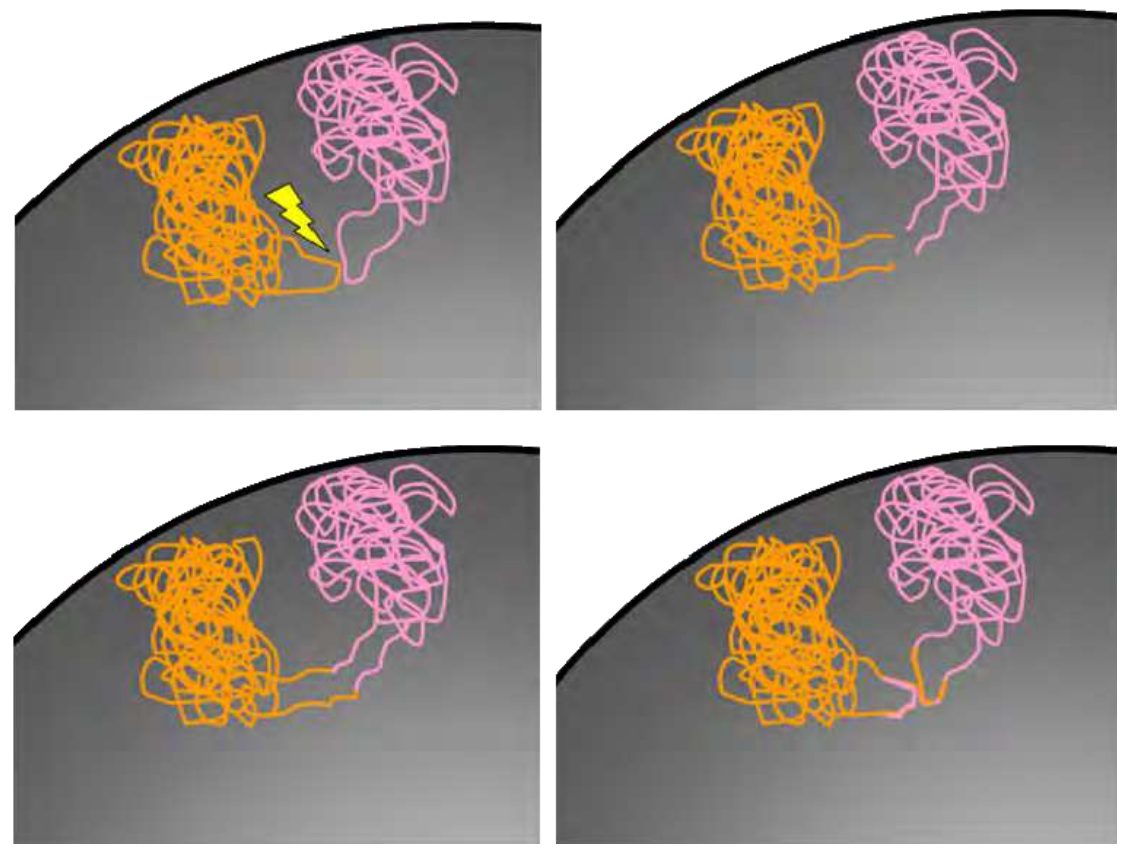

Fig. 3. Spatial organization of chromosomes in the formation of translocations after irradiation. Neighbouring CTs (yellow and red) are impaired by radiation, and closely juxtaposed DSBs created free chromosome ends. These ends could improperly join and formed a chromosome translocation by recombination.

Interestingly, the positioning of the specific regions or gene loci of the two chromosomes is involved in the translocation event, which is another important fact in cancerous transformation. The $\mathrm{t}(9 ; 22)(\mathrm{q} 34 ; \mathrm{q} 11)$ translocation in chronic myeloid leukaemia results in the fusion of $\mathrm{BCR}$ and $\mathrm{ABL}$, and these genes are in closer spatial proximity in normal heamatopoetic cells than other, non-translocating gene pairs (Lukasova et al., 1997; Kozubek et al., 1997; Neves et al., 1997; as cited in Meaburn et al., 2007). Significantly, exposure to radiation, a known inducer of leukaemia, results in BCR and ABL locating nearer to each other, in a more central part of the nucleus (Lukasova et al., 1997; Kozubek et al., 1997, as cited in Meaburn et al., 2007). A translocation involving HSA15 and 17, and the 
consequential fusion of the PML and RARa genes, is common in promyeloctic leukemia. PML and RARa are recurrently found in close proximity in normal B cells at a higher rate than the control gene pairs (Neves et al., 1997). An equivalent correlation between the proximity of genes and translocation frequency is observed in Burkitt's lymphoma. Notably, in B-cells the spatial proximity between MYC and any one of its multiple translocation partners (IGH, IGL, or IGK) decreases as the clinically observed incidence of translocation for the given pair increases (reviewed in Meaburn et al., 2007). Proximity effects on translocation formation are not limited to neighbour chromosome territories nor gene loci located on different chromosomes, but also apply to events on the same chromosome. The RET and the H4 genes are frequent translocation partners in thyroid tumours, and they are both located on chromosome 10 about $30 \mathrm{MB}$ apart, but are brought together in the 3D space of the cell nucleus by the looping of the chromosome fibre; thus juxtapositioning and predisposing them to undergo a mutual translocation, resulting in an internal chromosome reversion (Nikiforova et al., 2000). Interestingly, translocation events could lead to heterochromatin-mediated gene silencing. Bártová et al. (2002) demonstrated that $t(X ; 13)$ contributes to RB1 gene (13q14) silencing, also involving DNA methylation in a gene copy close to the translocated chromatin mass of HAS X. The changes in RB1 gene expression play an important role in the progression of the retinoblastoma tumour.

\section{Conclusions}

Higher-order genomic organization, involving the structure of the chromatin fibre, epigenetic modifications of chromatin, chromatin loops, chromatin compaction, and chromosome territories is a fundamental element, but not for storage of genetic information and it's processing alone. As was described above, it is also the basis for the maintenance of genome stability and its integrity impaired by external and internal damaging agents, including ionizing radiation. Here, it was shown how the higher-order chromatin structure influences DSB induction, mobility, repair, and mis-repair. In addition, chromatin structural defects lead to a list of specific diseases. The function of global higher-order chromatin organization, which maintains genome integrity, is emerging as an integral mechanism in the pathological processes, such as the formation of cancer translocations. Elucidation of the precise role and molecular mechanisms involved in maintaining chromatin structure and its role will be essential for our understanding of these all-important events. The understanding of genome functional organization; also in the context of the nuclear architecture will, in the future, provide promising developments of novel diagnostics and therapeutic strategies.

\section{Acknowledgment}

This work was supported by the grant of Ministry of Interior: VG20102014001.

\section{References}

Albiez, H.; Cremer, M.; Tiberi, C.; Vecchio, L.; Schermelleh, L.; Dittrich, S.; Kupper, K.; Joffe, B.; Thormeyer, T.; von Hase, J.; Yang, S.; Rohr, K.; Leonhardt, H.; Solovei, I.; Cremer, C.; Fakan, S. \& Cremer, T. (2006). Chromatin domains and the 
interchromatin compartment form structurally defined and functionally interacting nuclear networks. Chromosome Research, Vol. 14, pp. 707-733, ISSN 0967-3849.

Ayoub, N.; Jeyasekharan, A.D.; Bernal, J.A. \& Venkitaraman, A.R. (2008). HP1- $\beta$ mobilization promotes chromatin changes that initiate the DNA damage response. Nature, Vol. 453, No. 7195, pp. 682-686, ISSN 0028-0836.

Ayoub, N.; Jeyasekharan, A.D.; Bernal, J.A. \& Venkitaraman, A.R. (2009a). Paving the way for H2AX phosphorylation: chromatin changes in the DNA damage response. Cell Cycle, Vol. 8, No. 10, pp. 1494-1500, ISSN 1538-4101.

Ayoub, N.; Jeyasekharan, A.D.; \& Venkitaraman, A.R. (2009b). Mobilization and recruitment of HP1: a bimodal response to DNA breakage. Cell Cycle, Vol. 8, No. 18, pp. 29452950, ISSN 1538-4101.

Bártová, E.; Kozubek, S.; Jirsová, P.; Kozubek, M.; Gajová, H.; Lukášová, E.; Skalníková, M.; Gaňová, A.; Koutná, I. \& Hausmann, M. (2002). Nuclear structure and gene activity in human differentiated cells. Journal of Structural Biology, Vol. 139, pp. 76-89, ISSN 1047-8477.

Bártová, E.; Krejčí, J.; Harničarová, A.; Galiová, G. \& Kozubek, S. (2008). Histone modifications and nuclear architecture: A review. Journal of Histochemistry $\mathcal{E}$ Cytochemistry, Vol. 56, No.8, pp. 711-721, ISSN 0022-1554.

Boyle, S.; Gilchrist, S.; Bridger, J.M.; Mahy, N.L.; Ellis, J.A. \& Bickmore, W.A. (2001). The spatial organization of human chromosomes within the nuclei of normal and emerinmutant cells. Human Molecular Genetics, Vol. 10, pp. 211-219, ISSN 09646906.

Branco, M.R. \& Pombo, A. (2006). Intermingling of chromosome territories in interphase suggests role in translocations and transcription-dependent associations. PLoS Biology, Vol. 4, e138, ISSN 1544-9173.

Cann, K.L. \& Dellaire, G. (2011). Heterochromatin and the DNA damage response: the need to relax. Biochemistry and Cell Biology, Vol.89, pp. 45-60, ISSN 0829-8211.

Chambeyron, S.; Da Silva, N.R.; Lawson, K.A. \& Bickmore, W.A. (2005). Nuclear reorganisation of the Hoxb complex during mouse embryonic development. Development, Vol. 132, pp. 2215-2223, ISSN 0950-1991.

Cowell, I.G.; Sunter, N.J.; Singh, P.B.; Austin, C.A.; Durkacz, B.W.; Tilby, M.J. \& Sullivan, B. (2007). $\gamma \mathrm{H} 2 \mathrm{AX}$ foci form preferentially in euchromatin after ionizing-radiation. PLoS One, Vol. 2, No. 10, e1057, ISSN 1932-6203.

Cremer, T. \& Cremer, C. (2001). Chromosome territories, nuclear architecture and gene regulation in mammalian cells. Nature Reviews Genetics, Vol. 2, pp. 292-301, ISSN 1471-0056.

Cremer, T.; Cremer, M.; Dietzel, S.; Muller, S.; Solovei, I. \& Fakan, S. (2006). Chromosome territories - a functional nuclear landscape. Current Opinion in Cell Biology, Vol. 18, pp. 307-316, ISSN 0955-0674.

Cremer, T. \& Cremer, M. (2010). Chromosome territories. Cold Spring Harbour Perspectives in Biology, Vol. 2, a003889, ISSN 1943-0264.

Dinant, C. \& Luijsterburg, M.S. (2009). The emerging role of HP1 in the DNA damage response. Molecular and Cellular Biology, Vol. 29, No. 24, pp. 6335-6340, ISSN 02707306.

Dietzel, S.; Jauch, A.; Kienle, D.; Qu, G.; Holtgreve-Grez, H.; Eils, R.; Munkel, C.; Bittner, M.; Meltzer, P.S.; Trent, J.M. \& Cremer, T. (1998). Separate and variably shaped 
chromosome arm domains are disclosed by chromosome arm painting in human cell nuclei. Chromosome Research, Vol. 6, No. 1, pp. 25-33, ISSN 0967-3849.

Dietzel, S.; Schiebel, K.; Little, G.; Edelmann, P.; Rappold, G.A.; Eils, R.; Cremer, C. \& Cremer, T. (1999). The 3D positioning of ANT2 and ANT3 genes within female X chromosome territories correlates with gene activity. Experimental Cell Research, Vol. 252, pp. 363-375, ISSN 0014-4827.

Dellaire, G.; Kepkay, R. \& Bazett-Jones, D.P. (2009). High resolution imaging of changes in the structure and spatial organization of chromatin, $\gamma-\mathrm{H} 2 \mathrm{AX}$ and the MRN complex within etoposide-induced DNA repair foci. Cell Cycle, Vol. 8, No. 22, pp. 3750-3769, ISSN 1538-4101.

Dundr, M.; Ospina, J.K.; Sung, M.H.; John, S.; Upender, M.; Ried, T.; Hager, G.L. \& Matera, G. (2007). Actin-dependent intranuclear repositioning of an active gene locus in vivo. Journal of Cell Biology, Vol. 179, No. 6, pp. 1095-1103, ISSN 0021-9525.

Falk, M. ; Lukasova, E. ; Gabrielova, B. ; Ondrej, V. \& Kozubek, S. (2007). Chromatin dynamics during DSB repair. BBA Molecular Cell Research, Vol. 1773, pp. 1534-1545, ISSN 0167-4889.

Falk, M.; Lukasova, E. \& Kozubek, S. (2008). Chromatin structure influences the sensitivity of DNA to $\gamma$-radiation. BBA Molecular Cell Research, Vol. 1783, pp. 2398-2414, ISSN 0167-4889.

Falk, M.; Lukasova, E. \& Kozubek, S. (2010). Higher order chromatin structure in DSB induction, repair and misrepair. Mutation Research, Vol. 704, pp. 88-100, ISSN 13835742.

Ferreira, J.; Paolella, G.; Ramos, C. \& Lamond, A.I. (1997). Spatial organization of large-scale chromatin domains in the nucleus: A magnified view of single chromosome territories. Journal of Cell Biology, Vol. 139, pp. 1597-1610, ISSN 0021-9525.

Fraser, P. (2006). Transcriptional control thrown for a loop. Current Opinion in Genetic Development, Vol. 16, pp. 490-495, ISSN 0950-1991.

Fraser, P. \& Bickmore, W. (2007). Nuclear organization of the genome and the potential for gene regulation. Nature, Vol. 447, pp. 413-417, ISSN 0028-0836.

Gilbert, N.; Boyle, S.; Fiegler, H.; Woodfine, K.; Carter, N.P. \& Bickmore, W.A. (2004). Chromatin architecture of the human genome: gene-rich domains are enriched in open chromatin fibers. Cell, Vol. 118, pp. 555-566, ISSN 0092-8674.

Goetze, S.; Mateos-Langerak, J.; Gierman, H.J.; de Leeuw, W.; Giromus, O.; Indemans, M.H.G.; Koster, J.; Ondrej, V.; Versteeg, R. \& van Driel, R. (2007). The 3D structure of human interphase chromosome is related to the transcriptome map. Molecular and Cellular Biology, Vol.27, pp. 4475-4487, ISSN 0270-7306.

Goodarzi, A.A. ; Noon, A.T. ; Deckbar, D. ; Ziv, Y. ; Shiloh, Y. ; Lobrich, M. \& Jeggo, P.A. (2008). ATM signaling facilitates repair of DNA double-strand breaks associated with heterochromatin. Molecular Cell, Vol. 31, pp. 167-177, ISSN 1097-2765.

Guelen, L.; Pagie, L.; Brasset, E.; Meuleman, W.; Faza, M.B.; Talhout, W.; Eussen, B.H.; de Klein, A.; Wessels, L.; de Laat, W. \& van Steelsen, B. (2008). Domain organization of human chromosomes revealed by mapping of nuclear lamina interactions. Nature, Vol. 453, pp. 948-951, ISSN 0028-0836.

Jenuwein, T. \& Allis, C.D. (2001). Translating the histone code. Science, Vol.293, pp. 10741080, ISSN 0036-8075. 
Kouzarides, T. (2007). Chromatin modifications and their function. Cell, Vol.128, pp. 693-705, ISSN 0092-8674.

Kruhlak, M.J.; Celeste, A.; Dellaire, G.; Fernandez-Capetillo, O.; Müller, W.G.; McNally, J.G.; Bazett-Jones, D.P. \& Nussenzweig, A. (2006). Changes in chromatin structure and mobility in living cells at sites of DNA double-strand breaks. Journal of Cell Biology, Vol. 172, No. 6, pp. 823-834, ISSN 0021-9525.

Kúpper, K.; Kőlbl, A.; Biener, D.; Dittrich, S.; von Hase, J.; Thormeyer, T.; Fiegler, H.; Carter, N.P.; Speicher, M.R.; Cremer, T. \& Cremer, M. (2007). Radial chromatin positioning is shaped by local gene density, not by gene expression. Chromosoma, Vol. 116, pp. 285-306, ISSN 0009-5915.

Luijsterburg, M.C.; Dinant, C.; Lans, H.; Stap, J.; Wienrnasz, E., Lagerwerf, S.; Warmerdam, D.O.; Lindh, M.; Brink, M.C.; Dobrucki, J.W.; Aten, J.A.; Fousteri, M.I.; Jansen, G.; Dantuma, N.P.; Vermeulen, W.; Mullenders, L.H.F.; Houtsmuller, A.B.; Verschure, P.J. \& van Driel, R. (2009). Heterochromatin protein 1 is recruited to various types of DNA damage. Journal of Cell Biology, Vol. 185, No. 4, pp. 577-586, ISSN 0021-9525.

Markova, E.; Schultz, N. \& Belyaev, I.Y. (2007). Kinetics and dose response of residual 53BP1/gamma-H2AX foci: co-localization, relationship with DSB repair and clonogenic survival. International Journal of Radiation Biology, Vol. 83, No. 5, pp. 319329, ISSN 0955-3002.

Mathas, S.; Kreher, S.; Meaburn, K.J.; Johrens, K.; Lamprecht, B.; Assaf, C.; Sterry, W. ; Kadin, M.E.; Daibat, M.; Joos, S.; Hummel, M.; Stein, H.; Janz, M.; Anagnostopoulos, I.; Schrock, E.; Misteli, T. \& Dörken, B. (2009). Gene deregulation and spatial genome reorganization near breakpoints prior to formation of translocations in anaplastic large cell lymphoma. Proceedings of the National Academyof Sciences of the USA, Vol. 106, No. 14, pp. 5831-5836, ISSN 0027-8424.

Meaburn, K.J. \& Misteli, T. (2007). Cell biology: Chromosome territories. Nature, Vol. 445, pp. 379-381, ISSN 0028-0836.

Meaburn, K.J.; Misteli, T. \& Soutoglou, E. (2007). Spatial genome organization in the formation of chromosomal translocations. Seminars in Cancer Biology, Vol. 17, No. 1, pp. 80-90, ISSN 1044-579X.

Misteli, T. (2007). Beyond the sequence: cellular organization of genome function. Cell, Vol. 128 , pp. 787-800, ISSN 0092-8674.

Misteli, T. (2010). Higher-order genome organization in human disease. Cold Spring Harbour Perspectives in Biology, Vol. 2, a000794, ISSN 1943-0264.

Molenaar, C.; Wiesmeijer, K.; Verwoerd, N.P.; Khazen, S.; Eils, R.; Tanke, H.J. \& Dirks, R.W. (2003). Visualizing telomere dynamics in living mammalian cells using PNA probes. EMBO Journal, Vol. 22, pp. 6631-6641, ISSN 0261-4189.

Múnkel, C. \& Langowski, J. (1998). Chromosome structure predicted by a polymer model. Physical Review E, Vol. 57, pp5888-5896, ISSN 1539-3755.

Nikiforova, M.N.; Stringer, J.R.; Blough, R.; Medvedovic, M.; Fagin, J.A. \& Nikiforov, Y.E. (2000). Proximity of chromosomal loci that participate in radiation-induced rearrangements in human cells. Science, Vol. 290, pp. 138-141, ISSN 0036-8075.

Ondrej, V.; Lukasova, E. ; Krejci, J. ; Matula, P. \& Kozubek S. (2008). Lamin A/C and polymeric actin in genome organization. Molecules and Cells, Vol. 26, pp. 356-361, ISSN 1016-8478. 
Parada, L.A.; McQueen, P.G. \& Misteli, T. (2004). Tissue-specific spatial organization of genomes. Genome Biology, Vol. 12, pp. 1692-1697, ISSN 1474-7596.

Parada, L.A. \& Misteli, T. (2002). Chromosome positioning in the interphase nucleus. Trends in Cell Biology, Vol. 12, No. 9, pp. 425-432, ISSN 0962-8924.

Pederson, T. (2000). Half a Century of "The Nuclear Matrix". Molecular Biology of the Cell, Vol. 11, No. 3, pp. 799-805, ISSN 1939-4586.

Sachs, R.; van den Engh, G.; Trask, B.J.; Yokota, H. \& Hearst, J.E. (1995). A randomwalk/giant-loop model for interphase chromosomes. Proceedings of the National Academyof Sciences of the USA, Vol. 92, pp. 2710-2714, ISSN 0027-8424.

Sadoni, N.; Langer, S.; Fauth, C.; Bernardi, G.; Cremer, T.; Turner, B.M. \& Zink, D. (1999). Nuclear organization of mammalian genomes. Polar chromosome territories build up functionally distinct higher order compartments. Journal of Cell Biology, Vol. 146, pp. 1211-1226, ISSN 0021-9525.

Shi, Y.; Lan, F.; Matson, C.; Mulligan, P.; Whetstine, J.R.; Cole, P.A.; Casero, R.A. \& Shi, Y. (2004). Histone demethylation mediated by the nuclear amine oxidase homolog LSD1. Cell, Vol.119, pp. 941-953, ISSN 0092-8674.

Trojer, P. \&Reinberg, D. (2007). Facultative heterochromatin: Is there a molecular signature. Molecular Cell, Vol. 128, pp. 1-12, ISSN 1097-2765.

Van Driel, R.; Fransz, P.F. \& Verschure, P.J. (2003). The eukaryotic genome: a system regulated at differenthierarchical levels. Journal of Cell Science, Vol. 116, pp. 40674075, ISSN 0021-9533.

Verschure, P.J.; van Der Kraan, I.; Manders, E.M. \& van Driel, R. (1999). Spatial relationship between transcription sites and chromosome territories. Journal of Cell Biology, Vol. 147, pp. 13-24, ISSN 0021-9525.

Visser, A.E.; Jaunin, F.; Fakan, S. \& Aten, J.A. (2000). High resolution analysis of interphase chromosome domains. Journal of Cell Science, Vol. 113, pp. 2585-2593, ISSN 00219533.

Volpi, E.V.; Chevret, E.; Jones, T.; Vatcheva, R.; Williamson, J.; Beck, S.; Campbell, R.D.; Goldsworthy, M.; Powis, S.H.; Ragoussis, J.; Trowsdale, J. \& Sheer, D. (2000). Largescale chromatin organization of the major histocompatibility complex and other regions of human chromosome 6 and its response to interferon in interphase nuclei. Journal of Cell Science, Vol. 113, pp. 1565-1576, ISSN 0021-9533.

Weierich, C.; Brero, A.; Stein, S.; von Hase, J.; Cremer, C.; Cremer, T. \& Solovei, I. (2003). Three-dimensional arrangements of centromeres and telomeres in nuclei of human and murine lymphocytes. Chromosome Research, Vol. 11, pp. 485-502, ISSN 09673849.

Williams, R.R.; Broad, S.; Sheer, D. \& Ragoussis, J. (2002). Subchromosomal positioning of the epidermal differentiation complex (EDC) in keratinocyte and lymphoblast interphase nuclei. Experimental Cell Research, Vol. 272, pp. 163-175, ISSN 0014-4827.

Woodcock, Ch.L. \& Ghosh, R.J. (2010). Chromatin higher-order structure and dynaics. Cold Spring Harbour Perspectives in Biology, Vol. 2, a000596, ISSN 1943-0264.

Yokota, H.; van den Engh, G.; Hearst, J.E.; Sachs, R. \& Trask, B.J. (1995). Evidence for the organization of chromatin in megabase pair-sized loops arranged along a random walk path in the human G0/G1 interphase nucleus. Journal of Cell Biology, Vol. 130, No. 6, 1239-1249, ISSN 0021-9525. 
Zarebski, M.; Wiernasz, E., \& Dobrucki, J.W. (2009). Recruitment of heterochromatin protein 1 to DNA repair sites. Cytometry A, Vol. 75, No. 7, pp. 619-625, ISSN 1552-4922.

Zhang, R.; Liu, S.T.; Chen, W.; Bonner, M.; Pehrson, J.; Yen, T.J. \& Adams, P.D. (2007). HP1 proteins are essential for a dynamic nuclear response that rescues the function of perturbed heterochromatin in primary human cells. Molecular and Cellular Biology, Vol. 27, No. 3, pp. 949- 962, ISSN 0270-7306. 


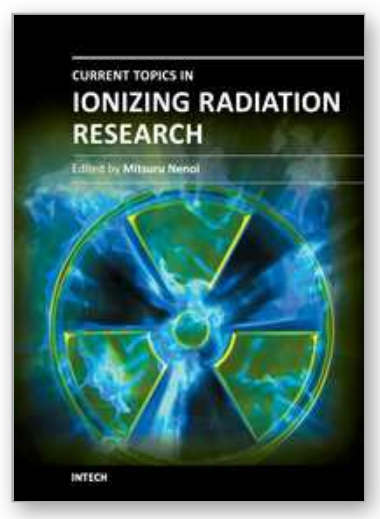

\author{
Current Topics in lonizing Radiation Research \\ Edited by Dr. Mitsuru Nenoi
}

ISBN 978-953-51-0196-3

Hard cover, 840 pages

Publisher InTech

Published online 12, February, 2012

Published in print edition February, 2012

Since the discovery of $X$ rays by Roentgen in 1895 , the ionizing radiation has been extensively utilized in a variety of medical and industrial applications. However people have shortly recognized its harmful aspects through inadvertent uses. Subsequently people experienced nuclear power plant accidents in Chernobyl and Fukushima, which taught us that the risk of ionizing radiation is closely and seriously involved in the modern society. In this circumstance, it becomes increasingly important that more scientists, engineers and students get familiar with ionizing radiation research regardless of the research field they are working. Based on this idea, the book "Current Topics in lonizing Radiation Research" was designed to overview the recent achievements in ionizing radiation research including biological effects, medical uses and principles of radiation measurement.

\title{
How to reference
}

In order to correctly reference this scholarly work, feel free to copy and paste the following:

Vladan Ondřej (2012). Genome Integrity and Organization in the Context of Radiobiology, Current Topics in Ionizing Radiation Research, Dr. Mitsuru Nenoi (Ed.), ISBN: 978-953-51-0196-3, InTech, Available from: http://www.intechopen.com/books/current-topics-in-ionizing-radiation-research/genome-integrity-andorganization-in-the-context-of-radiobiology

\section{INTECH}

open science | open minds

\author{
InTech Europe \\ University Campus STeP Ri \\ Slavka Krautzeka 83/A \\ 51000 Rijeka, Croatia \\ Phone: +385 (51) 770447 \\ Fax: +385 (51) 686166 \\ www.intechopen.com
}

\author{
InTech China \\ Unit 405, Office Block, Hotel Equatorial Shanghai \\ No.65, Yan An Road (West), Shanghai, 200040, China \\ 中国上海市延安西路65号上海国际贵都大饭店办公楼 405 单元 \\ Phone: +86-21-62489820 \\ Fax: $+86-21-62489821$
}


(C) 2012 The Author(s). Licensee IntechOpen. This is an open access article distributed under the terms of the Creative Commons Attribution 3.0 License, which permits unrestricted use, distribution, and reproduction in any medium, provided the original work is properly cited. 